\title{
LA ARETÉ COMO KÁTHARSIS EN EL FEDÓN. FORMULACIÓN Y PROYECCIONES ÉTICAS EN LA OBRA DE PLATÓN*
}

\author{
Daniel Brito García \\ Universidad Católica de la Santísima Concepción. \\ Concepción. Chile
}

Resumen: El presente estudio pretende destacar los aspectos éticos contenidos en el Fedón, al centrarse en la conceptualización de la virtud ascética y su lugar en el diálogo. A su vez, se destaca cómo la virtud ascética se vincula con la kátharsis del alma y se proyecta una posible continuidad en los planteamientos morales del Fedón, con respecto a la exposición y desarrollo de las virtudes en la República.

Palabras claves: Alma - cuerpo - hombre - areté - kátharsis

\section{THE ARETÉ AS KÁTHARSIS IN THE PHAEDO. FORMULATION AND ETHICS PROJECTIONS IN THE WORK OF PLATO}

Abstract: The present study aims to highlight the ethical content in the Phaedo, to focus on the conceptualization of ascetic virtue and its place in the dialogue. Furthermore, highlights how the ascetic virtue is linked to the katharsis of soul and a possible continuity extends moral approaches in the Phaedo, with respect to exposure and development of virtues in the Republic.

Key words: soul - body - man - areté - kátharsis

Recibido: 29.12.2013 - Aceptado: 7.03.2014

Correspondencia: DANIEL BRITO GARCÍA debrito@filosofia.ucsc.cl

Universidad Católica de la Santísima Concepción,

Campus San Andrés, Alonso de Ribera 2850, Concepción. Fono: 57758442.

* Las ideas fundamentales del presente trabajo fueron expuestas en la comunicación brindada bajo el marco del III Congreso nacional de Filosofía, realizado en Valparaíso, entre los días 4 al 8 de noviembre de 2013. 


\section{T ntroducción. La problemática moral en el Fedón.}

Irrefutable es el hecho que la problemática del alma bajo tratamiento platónico ha logrado una influencia inconmensurable no sólo para sus contemporáneos, sino también para la posteridad de la tradición filosófica. Dicho tópico constantemente se ha presentado desde la relación con la moral platónica (el más claro ejemplo es la República, pieza capital de la ética de Platón), siendo la excepción a la regla el caso del Fedón. ¿Por qué sucede esto? Probablemente la respuesta más próspera sea que en un primer acercamiento a un diálogo como Fedón, que además presenta como subtítulo 'o del alma', se tiende justamente a explorar una lectura de corte metafísica - antropológica con matices de religiosidad mística. Es más; el acento que se le ha dado al diálogo ha tenido diversos matices: Bluck (1955, p. 1 - 2) manifiesta que la teoría de las Formas y la teoría de la inmortalidad del alma son interdependientes en el Fedón, con lo que Platón tendría por motivación justificar su creencia en ambas como objeto primero del diálogo; Hackforth (1955, p. 3 - 7) plantea que el objeto esencial del Fedón no radica en probar que el alma es inmortal; extremando la medida, Dixsaut (1991, p. 2 - 3) señala que dicha demostración, de hecho, no se alcanza; Gadamer (1980, p. 22) es enfático en sostener que el ateniense posee real conciencia de que los argumentos a favor de la inmortalidad del alma no son definitorios, sino sólo expresiones de una presunción; y Guthrie (1998, p. 352) concluye su estudio del diálogo expresando que Platón estaría convencido de la verdad de ciertas cuestiones, pero no dispuesto a ofrecer una completa prueba dialéctica.

Lo que debería llamarnos la atención sobre esto es que si bien el diálogo propone un claro énfasis en la problemática del alma, las pruebas de su inmortalidad y sus fines últimos, lo hace a través de un camino claramente ético.

En vista de esto, por un lado, nuestra tarea radica en esclarecer la visión ascética ${ }^{1}$ que el discípulo de Sócrates manifiesta en ciertos aspectos de su reflexión

${ }^{1}$ El término 'ascético' proviene de la raíz griega ỏokéc que originariamente tenía el sentido del cómo llevar a cabo un trabajo; una 'forma de realizar un trabajo'. Es uno de los derivados 
moral, y por otro, establecer el vínculo que posee la virtud y la purificación del alma, a partir de las claves éticas entregadas por Platón en el Fedón. Bajo estas premisas, cabe preguntarse ¿qué tiene el Fedón que resulta ser la fuente ineludible para nuestra investigación? Primero, en él se encuentran ciertos elementos morales que si bien están presentes en el texto han tenido poca atención por parte de los especialistas, especialmente lo que atañe a la exposición de la virtud ascética; segundo, en dicho diálogo no es explícita la vinculación de la moral con el alma, y; tercero, resulta factible analizar la posible continuidad de la teoría moral platónica, al menos en el caso de la República.

Además, nos centraremos en este diálogo para exponer cómo se logra purificar el alma del hombre a partir de la virtud, puesto que revela los aspectos centrales del pensamiento platónico en su época de madurez, como lo son las nociones de Idea, alma, conocimiento, inteligencia, participación, presencia, virtud, entre otras ${ }^{2}$. Teniendo presente esto, nos embarcaremos en la tarea de dilucidar, como objeto primero de nuestra exposición, el cómo la areté resulta ser, en sentido estricto, un rito kathártico para el hombre platónico sujeto a las cadenas corporales.

Para lograr este objetivo, y con el fin de dar coherencia a nuestro discurso, desarrollaremos el siguiente plan de trabajo: en un primer momento, analizaremos la relación entre kátharsis y thánatos, destacando la figura del filósofo y su radical ascetismo; en una segunda instancia, perfilaremos la areté como actividad kathártica del filósofo con el fin de acceder al conocimiento pleno de la realidad; en tercer lugar, mostraremos de qué manera la phrónesis se presenta como el rito kathártico y areté por antonomasia del quehacer filosófico; en cuarto lugar, visualizaremos la estrecha unión entre el alma, la virtud y el fin escatológico; y terminaremos otorgando algunas conclusiones sobre el problema tratado.

del término, ǒ $\sigma \kappa \eta \sigma \iota \varsigma$, que alude a la idea de entrenamiento con vistas a estar en forma para determinados ejercicios atléticos, la acepción corporal que se propagó hasta abarcar un contenido espiritual; es decir, un entrenamiento para fines espirituales. Platón emplea el término en sus

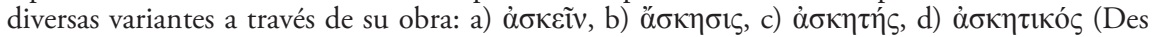
Places, 1964, p. 80). Curiosamente, en el Fedón no hay la más mínima referencia explícita de dicho término, aun cuando el ascetismo es una nota indiscutidamente característica del diálogo. Cf. Chantraîne (1968-77, p. 124) para ver la evolución etimológica del término.

2 Theodor Gomperz (2000, p. 443) afirma al respecto: “...el valor del Fedón como obra artística de filosofía es inmenso. En efecto, en ella se congregan todos los méritos de Platón, todo aquello que adorna sus obras restantes, sin ninguna de las exageraciones que desfiguran muchas de ellas. El diálogo está en un punto de equilibrio del desarrollo platónico". 


\section{1.- Kátharsis y Thánatos. La figura del filósofo y el ascetismo platónico del Fedón.}

El Fedón, diálogo marcado por la fusión de horizontes de una importante tradición religiosa y la apertura a la ciencia propia de la época (Gadamer; 1980, p. $21 ; 1999$ (a) , p. 48) ${ }^{3}$, perfila la figura del filósofo como aquel que espera, se esfuerza

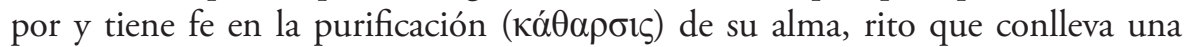
preparación adecuada para la muerte $(\theta \alpha ́ v \alpha \tau o \zeta)^{4}$, entendida como la separación del alma con respecto al cuerpo (Fedón, 64 c) ${ }^{5}$; la separación del elemento divino respecto de lo humano (Fedón, 80 a). Es aquel que se ejercita en morir y para él estar muerto es a lo que menos debe temer un hombre (Fedón, 67 e).

La descripción contenida en la figura del filósofo del Fedón nos recuerda la clásica alusión proveniente de la corriente órfica - pitagórica que alude al cuerpo como prisión, cárcel, tumba o sepulcro del alma, idea que también se asoma en otros diálogos platónicos ${ }^{6}$, siendo una de las claves intelectuales heredada de la tradición que sustenta el pensamiento sobre el hombre en nuestro autor ${ }^{7}$. En palabras de Platón, "los humanos estamos en una especie de prisión ( $\varphi \rho 0 v \rho \tilde{\alpha})$ " (Fedón, 62 b) que nos coarta frente al anhelo propio de la desligación del cuerpo con vistas al ascenso celeste. Para Sócrates, es lógico que un hombre que de verdad

${ }^{3}$ Cf. Flavia Gilda Gioia (2009; p. 6).

${ }^{4}$ Cornford (2004, p. 246), refiriéndose al discurso del Fedón concerniente a la declaración de la visión pitagórica de la vida y la muerte, subraya que para Platón ésta se debe tomar más bien como una expresión de $f e$ que una teoría establecida a través de un argumento. Es un mythos, no

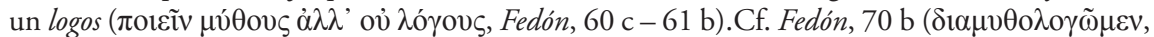
traducible por 'que contemos cuentos' o 'conversar'). No obstante su visión, el propósito del diálogo es pasar de la creencia popular al logos y a las pruebas de la inmortalidad del alma expuestas en él.

${ }^{5}$ En el Fedón se presentan tres concepciones sobre la muerte: la presente, la contenida en el pasaje $91 \mathrm{~d}$, donde la muerte se entiende como la "destrucción del alma", y una tercera que encontramos en el pasaje 107 c, donde es concebida como "la disolución del todo".

${ }^{6}$ Cf. Gorgias 493 a 3, en que dicha idea se expone con el termino $\sigma \tilde{\eta} \mu \alpha$, traducido como 'sepulcro', haciendo clara alusión al cuerpo $(\sigma \tilde{\omega} \mu \alpha)$; Crátilo 400 c 1 , en que ésta 'tumba' nuevamente se expresa con la misma palabra griega, y que líneas siguientes se presenta bajo el rotulo de 'prisión', ahora con el término $\delta \varepsilon \sigma \mu \omega \tau \eta \rho i ́ o v$. Véase Crátilo 404 c para bosquejar la etimología del término cuerpo dada por Platón.

${ }^{7} \mathrm{Si}$ bien el Fedón desarrolla en su entramado más espeso argumentos en orden a demostrar la inmortalidad del alma, metodológicamente el diálogo está atravesado por la tradición popular y religiosa que la pluma platónica nos heredó. Goldschmidt, en su aún valioso trabajo Les Dialogues de Platon: Structure et Méthode dialectique (1971, p. 183-212), realiza el análisis metodológico del Fedón, y confirma que el diálogo parte de la tradición popular (Image), sube a la reflexión de las pruebas de la inmortalidad del alma (al nivel de Essence), y finaliza volviendo a la tradición religiosa con el mito escatológico. 
ha dedicado su vida a la filosofía y que, por tanto, se ha volcado a la reflexión en torno a la existencia humana, estando en trance de morir, tenga valor y esté bien esperanzado de que una vez que llegue al lugar celeste con los dioses va a obtener los mayores bienes, la contemplación eidética: visión verdadera de la realidad. Así es como la muerte será pronto considerada como una liberación, resultado directo de una purificación del alma respecto al cuerpo y a sus impedimentos, provocada a partir de la escisión de cuerpo y alma, con lo que supone Platón que ésta (el alma) subsiste en sí misma, aun cuando en la República necesite del cuerpo para concretar las acciones del rey-filósofo.

De acuerdo a lo anterior, el alma se manifiesta como el principio de vida ${ }^{8}$, como principio de movimiento (tal como se expone en el Fedro, $245 \mathrm{~d}-\mathrm{e}$ ), como lo racional y lo espiritual en el hombre, frente al cuerpo que se presenta como un mero instrumento y receptorio sensible. Así, los apetitos y deseos parecen quedar asignados únicamente al cuerpo ${ }^{9}$, mostrando una natural aunque férrea actitud ascética, como destaca Jaeger (2006, p. 563), expresada en el accionar virtuoso del filósofo, expuesta principalmente bajo la presentación del Fedón, manifiesto del ascetismo místico-religioso del autor ateniense.

Así es como en la figura del ascético filósofo la ocupación no se centra en el cuerpo, sino que, en cuanto puede, está apartado de éste, volcándose incesantemente hacia el alma. El filósofo aspira a liberar su alma al máximo de su triste vinculación con el cuerpo, avanzando hacia la plenitud del saber fuera de él, tal como, en forma análoga, la alcanza el hombre que logra salir y superar la situación cavernaria de la República (VII, 514 a y ss.). De esta manera, el alma del filósofo avanza desde la purificación hacia la liberación de ésta, y deja atrás las cadenas del cuerpo, tal como en el Fedón, y al igual que en la República, donde el prisionero de la caverna que logra escapar y deshacerse de las cadenas de la ignorancia, alcanza a contemplar, gradualmente, el conocimiento y la verdad ${ }^{10}$.

${ }^{8}$ Con respecto a la etimología del termino psyché, cf. Crátilo 399 d - 400 b. Para ahondar en la actividad etimológica desarrollada en el Crátilo, véase el trabajo de Adalbert Steiner, "Die Etymologien in Platons Kratylos", Archiv für Geschichte der Philosophie (1916), vol. 29, № 2, p. 109 - 132. Para un estudio sobre el origen de la divinidad del alma y de cómo ésta va conformando su significación originaria, véase Jaeger (2003, p. 77 - 92).

${ }^{9}$ Es curioso contrastar cómo en un diálogo de la talla de Fedón se manifiesta un ascetismo intelectual radical versus la perspectiva que nos entrega el erótico Banquete y su acento en el amor y el deseo. Para ahondar en esta radical diferencia véase G. M. A. Grube (2010, p. 203). Cf. República (437 b - 438 a), el alma concupiscible o apetitiva.

${ }^{10}$ Cf. Fedón, 67 c - d, tal como es expuesto más adelante. Lúcidamente el profesor Oscar Velásquez destaca esta situación en su artículo 'A $A \eta \dot{n} \theta \varepsilon 1 \alpha$ y sus usos y significados en Platón', Revista Iter, ¿Quid est veritas? (2005, p. 6), haciendo hincapié en el lenguaje sensorial de la similitud: “... 
De esta forma, Platón destaca que en la reflexión del filósofo se hace evidente el conocimiento de lo real, más que en cualquier otro momento. Eso sí, el alma reflexiona cuando no le perturba ninguna de esas cosas que se muestran en la sensación: ni el oído, ni la vista, ni el dolor, ni placer alguno, sino que ella se encuentra al máximo en sí misma mandando de paseo al cuerpo, y sin comunicarse ni adherirse a él ${ }^{11}$. Sólo así tiende hacia lo que existe realmente, con lo cual queda demostrado el antihedonismo ético propuesto por Platón, por lo menos en este diálogo ${ }^{12}$.

\section{2.- La Areté como actividad kathártika del filósofo para acceder al conocimiento.}

En este marco (Fedón, 65 d), Platón introduce, curiosamente a simple vista, la pregunta por la justicia como virtud, desde la cual se adentrará en la relación que posee la virtud con el filósofo y su limpieza del cuerpo. Sócrates interroga a Simmias si es que existe algo justo en sí o nada y, a su vez, si existe algo bello y bueno, obteniendo de esta manera la oportunidad de descubrir la flaqueza de su argumento. "¿Es que ya has visto alguna de tales cosas con tus ojos nunca?” (Fedón, 65 d) pregunta Sócrates, haciendo alusión a la teoría de las Ideas, que no pueden verse con los ojos del cuerpo, pero sí con el ojo del alma ( $\tau$ ò $\tau \tilde{\eta} \varsigma$

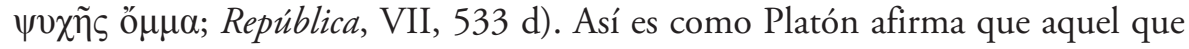
se prepara a pensar mejor y más exactamente cada cosa en sí de las que examina, llegaría lo más cerca posible al conocimiento de dichas cosas (Fedón, 65 e - 66 a).

En este sentido, se propone una concordancia entre el conocimiento y el alma humana y, a su vez, entre la ignorancia y el cuerpo. Por tanto, mientras tengamos el cuerpo y nuestra alma esté contaminada por la ruindad de éste,

verdad es algo apropiado al alma; y si según el Fedón el cuerpo es considerado un impedimento

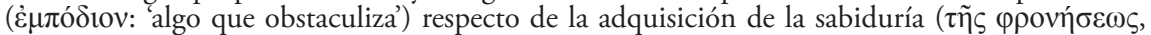
65 a), este no garantiza la verdad que mediante él se pudiera obtener. Hay en consecuencia una visión espiritual, que se continúa expresando en un lenguaje que tiene mucho de sensorial. Así, los que deben volver a la Caverna después de haber salido de ella, una vez que se habitúen a la oscuridad, verán (ő $\psi \varepsilon \sigma \theta \varepsilon$, Rep. 520 c) mil veces mejor que los de allí. Esta acción supone el uso de la vista para mirar, pero es una visión que se entiende que es realizada por almas que han experimentado el periplo completo de la Caverna”.

${ }^{11}$ A propósito de esto, Jaeger (2006, p. 563) destaca la visión del filósofo del Fedón confrontándola con la expuesta en el Banquete y la República.

${ }^{12}$ Si bien Platón está abiertamente en contra del hedonismo en todas sus aristas (al menos hasta Fedón), ya en República se da el balance entre lo que tiende al placer (o sea, la parte concupiscible del alma, aun cuando ésta se vea sometida a los mandatos de la parte racional) y el saber en el alma como armonía, y en Filebo (65 a y ss.) abraza el placer como complemento necesario para la vida del hombre. 
jamás se conseguirá suficientemente aquello que deseamos: la verdad y el saber

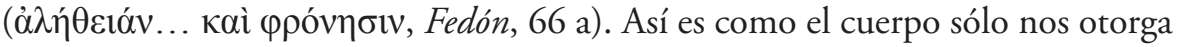
preocupaciones y complicaciones para obtener la sabiduría. Con esto, se logra demostrar que si alguna vez vamos a saber algo limpiamente, hay que separarse del cuerpo y hay que observar los objetos reales en sí con el alma por sí misma.

Así y sólo así obtendremos la sabiduría, objeto anhelado por sus amantes

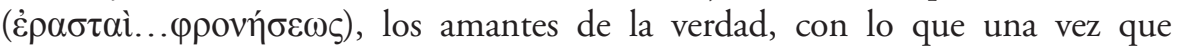
hayamos muerto $(\tau \varepsilon \lambda \varepsilon v \tau \eta ́ \sigma \omega \mu \varepsilon v)$, según nos muestra el ateniense, cumpliremos

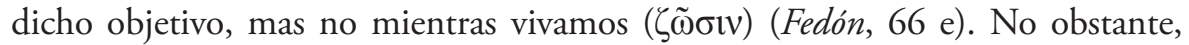
mientras vivamos estaremos más cerca del saber ( $\varepsilon i \delta \varepsilon ́ v \alpha l)$ en la medida en que no

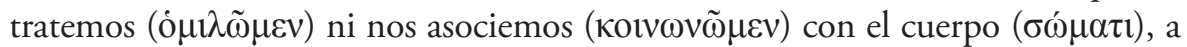
no ser en la estricta necesidad, y no nos contaminemos de la naturaleza suya, sino

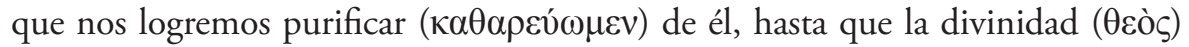

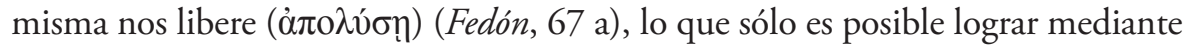
la práctica de la virtud, práctica eminentemente purificadora y sanadora, a través de la cual el filósofo se desprende de las ataduras del cuerpo, higienizando su alma.

Léon Robin (1935; p. 156 - 157) se pregunta cuál es la esencia de la virtud, y alude a que ya en el Fedón se da una respuesta: la del ascetismo. Esta respuesta es, fundamentalmente, que la virtud del hombre se da en una existencia extremadamente espiritual y liberada, en la medida de lo posible, de los requisitos y restricciones del cuerpo. Por lo tanto, para Platón, la gran apuesta de la vida es 'la práctica de morir'; el verdadero propósito de la vida humana es precisamente el final de ésta, teniendo presente siempre que la decisión arbitraria de quitarnos la vida (el suicidio) no es el camino para la contemplación de las Ideas, puesto que se debe estar al alero de la voluntad de los dioses. Este ideal es la 'purificación', y esto es lo que Platón llama 'tener preocupación por su alma', para Robin.

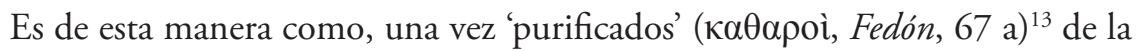
insensatez del cuerpo, estaremos en compañía de lo semejante y conoceremos por nosotros mismos todo lo puro, puesto que es eso lo verdadero, con lo que se logra una identidad entre ambas nociones. De hecho, Platón enfatiza aún más esto: "al que no esté puro me temo que no le es lícito captar lo puro" (Fedón, 67 b). Es más; en Fedón, 79 d, Platón nos señala que el alma "cuando examina sola y

${ }^{13}$ En esta cita me acojo a la traducción de Conrado Eggers Lan (EUDEBA, 1976, p. 109) por ser más cercana al sentido que aquí tiene el término $\kappa \alpha \theta \alpha \rho o i ̀$, siendo éste el de 'puro', 'limpio', más que el de 'desprendidos' como apuesta la traducción de García Gual. Cf. Fedón, 80 e, 82 b. 


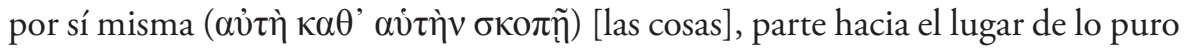

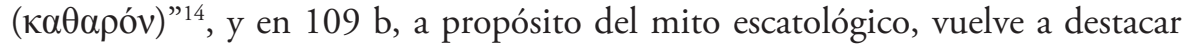
la pureza de los lugares a los que se debe dirigir el alma: "la tierra misma, en su

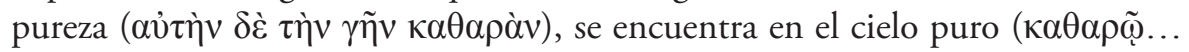

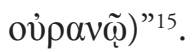

De lo anterior, se está en buen pie para la muerte cuando se tiene preparada la inteligencia y, a la vez, ésta está purificada, lo cual se logra a través de la virtud concretada en el accionar del filósofo. Platón, haciéndose cargo de lo mencionado, nos propone una definición de lo que entiende por purificación

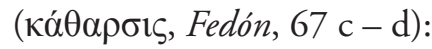

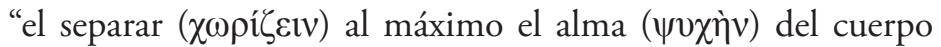
( $\sigma \omega ́ \mu \alpha \tau \sigma \zeta)$ y el acostumbrarse ella a recogerse y concentrarse en sí misma $^{16}$ fuera del cuerpo, y a habitar en lo posible, tanto en el tiempo presente como en el futuro, sola en sí misma ( $\mu$ óvๆv $\kappa \alpha \theta^{\text {' }}$ $\alpha v i \eta ́ v)$, liberada del cuerpo como de unas cadenas $(\delta \varepsilon \sigma \mu \tilde{\omega} v)$ ”.

Desde la perspectiva contemplativa, especial dedicación presto Festugière (1975, p. 123 y ss.) a la noción de 'purificación' inmersa en el Fedón y a su importancia dentro de la filosofía. Basándose en las Leyes, IV, 716 c - 717 a, destaca la relación del hombre con lo divino a través de 'purificaciones', y afirmándose, además, en Eurípides y su Ion (1315 - 1316) señala:

"Dios y todo lo que es Dios es puro. Por consiguiente, no puede uno aproximársele, no se puede complacer a lo divino más que a condición de participar de esa pureza. La katharótes del objeto exige en el sujeto un estado análogo. Si éste no la posee aún, debe necesariamente katharizesthai. Tal parece haber sido la génesis de la kátharsis platónica" (1975, p. 127).

Eggers Lan propone que esta 'génesis' del término ha sido justamente al modo inverso: "Platón piensa que al hombre se le exige la purificación por ser lo divino puro", acentuando la discusión (EUDEBA, 1976, p. 107, nota $\mathrm{N}^{\circ} 52$ ).

En definitiva, el filósofo busca liberar el alma del cuerpo y, con esto, la muerte toma una connotación positiva (Gorgias, 485 a, 499 c) en tanto separación y

${ }^{14}$ Cf. Fedón, 80 d, 83 e, República, IX, 572 a, 585 b.

${ }^{15}$ Cf. Fedón, 109 d, 114 c, República, VII, 520 d. 
liberación del alma por parte del yugo corporal, pudiendo acceder así a las Ideas suprasensibles. De acuerdo al relato de Sócrates, el filósofo en ningún otro lugar conseguirá de modo puro la sabiduría sino en el Hades ${ }^{17}$, o como señala en otro

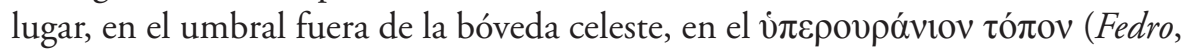
247 c 2).

\section{3.- La phrónesis como rito kathártico y areté fundamental del filósofo.}

Teniendo presente lo anterior, Sócrates pregunta a Simmias (Fedón, 68 c) si es que no se aplica especialmente la valentía a los que presentan la disposición de ánimo (propia del filósofo) y la temperancia, o incluso aquello que la gente llama popularmente temperancia ${ }^{18}$, o le es propio a quienes en grado extremo se despreocupan del cuerpo y viven dedicados a la filosofía. Es en este momento que a Sócrates le interesa resaltar que las verdaderas virtudes sólo las practica conscientemente el sabio, mientras que las demás se atienen a meras apariencias

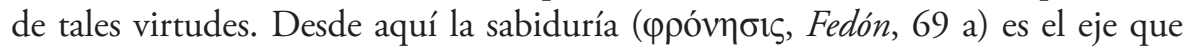
debe guiar nuestros actos ${ }^{19}$, y en base a aquélla se da la posibilidad de que existan

${ }^{16}$ La cursiva es mía. A propósito de la preparación de la inteligencia, Jaeger (2006, p. 563) destaca que "el saber se describe aquí [en el Fedón] como la 'concentración' del alma - uno de los conceptos psicológicos inmortales de Platón -, por medio de la cual ésta se sobrepone a la dispersión de los sentidos, que la empujaban siempre hacia el mundo exterior, para condensarse en su más genuina actividad interior". Jaeger, en base a esto, concluye que "en esta obra encuentra su expresión más acusada el antagonismo entre la naturaleza espiritual y sensorial del hombre". Se da la unidad en el pensamiento y su vínculo con las Ideas versus la multiplicidad de la realidad mutable. Véase Fedón, 83 a, donde se hace alusión al saber en los mismos términos.

${ }^{17}$ Platón cuando habla del Hades ("Aiðov, Fedón 80 c), hace alusión al sentido primitivo del término

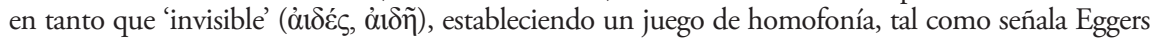
en su traducción del Fedón (EUDEBA, 1976, p.10, nota n¹17). Dicho sentido es el utilizado en este estudio. Cf. Crátilo, 404 b, en donde se da un sentido absolutamente distinto al aquí presentado.

${ }^{18}$ Fedón, 68 c: "el no dejarse excitar por los deseos, sino dominarlos moderada y ordenadamente".

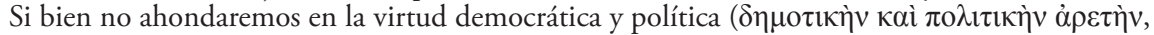
Fedón, 82 a - b), expuesta en Fedón 68 b - 69 a, propia de los amigos del cuerpo ( que, como plantea Platón, seguramente también son amigos de las riquezas ( $\varphi$ ı $\chi \propto \rho \eta ́ \mu \alpha \tau o \varsigma)$ y los honores ( $\varphi$ i ó $\imath \mu \rho \varsigma$, Fedón, 68 c), sujeta a la moralidad convencional de los sofistas, es necesario destacar que para los 'no-filósofos' la muerte es un mal (Fedón, $68 \mathrm{~d}-\mathrm{e}$ ), por lo que en base al temor de mayores males los valientes afrontan la muerte cuando la afrontan, o sea, por miedo y por temor son valientes todos menos los filósofos, al igual que los moderados: se han hecho moderados por su intemperancia. Véase Fedón, 82 a - b, República, VI, 500 d - c, y Leyes, IV, 710 a. Cf. W. K. C. Guthrie (1998, p. 330).

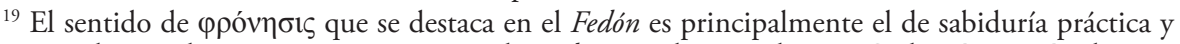
moral, no sólo teórica y técnica como la sophía. Hecho que destaca Carlos García Gual en su traducción de Fedón (Editorial Gredos, 2008, p. 49, nota pie p. 32), haciendo alusión a pasajes del diálogo como 66 e, 68 a y b. 
la verdadera valentía, moderación, justicia y, en la totalidad, la verdadera virtud

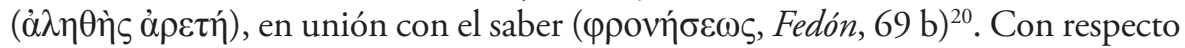
a lo anterior, Platón pone en boca de Sócrates lo siguiente (Fedón, 69 b - c):

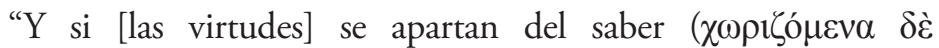
$\varphi \rho о v \eta ́ \sigma \varepsilon \omega \varsigma)$ y se truecan unas por otras, temo que la virtud resultante no sea sino un juego de sombras, y servil en realidad, y que no tenga nada sano ni verdadero. Acaso lo verdadero ( $\dot{\alpha} \lambda \eta \theta \dot{\varepsilon} \varsigma)$, en realidad, sea una cierta purificación (кá $\theta \alpha \rho \sigma i ́ s)$ de todos esos sentimientos, y también la moderación y la justicia y la valentía, y que la misma

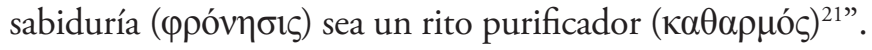

De aquí en adelante queda establecida la supremacía de la sabiduría hasta el final de la obra de Platón con respecto al orden y clasificación de las demás virtudes $^{22} \mathrm{y}$, además, se deja constancia, de manera contundente, de que el ejercicio de la virtud, representada a través de su caballo de batalla, en este caso la sabiduría, es el único rito purificador viable para una vida plena, sabia y feliz. Flavia Gilda Gioia (2009; p. 3) sintetiza plenamente este sentir:

"El Fedón es, pues, una exhortación a la sabiduría (phrónesis) ${ }^{23}$ que, en tanto examen permanente de la razón, se presenta como un

${ }^{20}$ Recordemos que la formulación del filósofo-rey en la República también tiende a destacar la urgencia del saber, pero ahora en clave social, orientada a dirigir los asuntos del Estado, aun cuando el propio Estado sea una proyección del hombre, específicamente del alma del hombre. En el Politico el acento se vislumbra desde la ciencia política.

${ }^{21}$ Cf. Fedón 82 d, en que se destaca el vínculo absoluto del rito purificador (katharmós) con la filosofía: "Así que entonces mandando de paseo todo eso ["las pasiones del cuerpo", $82 \mathrm{c}$ ], Cebes, aquellos a los que les importa algo su propia alma ( $\tau \tilde{\eta} \varsigma \dot{\varepsilon} \alpha v \tau \tilde{\omega} v \psi v \chi \tilde{\eta} \varsigma)$ y que no viven amoldándose al cuerpo $(\sigma \omega ́ \mu \alpha \tau \imath)$, no van por los mismos caminos que estos que no saben a dónde se encaminan, sino que considerando que no deben actuar en sentido contrario a la filosofía $\left(\varphi 1 \lambda\right.$ $\left.\lambda \sigma o \varphi i_{\alpha} \alpha\right)$ y a la liberación $(\kappa \alpha \theta \alpha \rho \mu \tilde{\omega})$ y el encanto de ésta, se dirigen de acuerdo con ella, siguiéndola por donde ella los guía ". Ésta cita y, a su vez, la cita de la que fue vinculada ésta, son los únicos dos pasajes en que se emplea el término katharmós al menos en lo que respecta al Fedón (Des Places, 1964, p. 270 - 271). Para revisar el sentido originario del término véase W. K. C. Guthie (1998, p. 254 - 255).

${ }^{22}$ En Fedón (69 a y ss.), se establece la superioridad de la sabiduría con respecto a las demás virtudes, estableciendo que en base a ella la valentía, la moderación y la justicia se sustentan. En

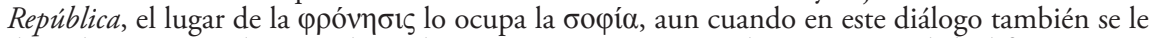
da un lugar preponderante a la inteligencia. En Leyes $(631 \mathrm{c}-\mathrm{d})$, a propósito de la diferenciación entre los bienes humanos y divinos, Platón ubica entre estos últimos, en el primer puesto, a la inteligencia ( $\varphi \rho o ́ v \eta \sigma ı)$; el segundo lugar lo asigna al estado prudente del alma acompañado de

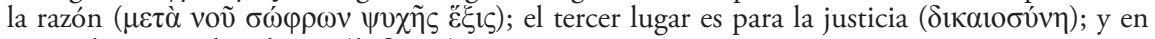

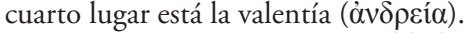

${ }^{23}$ Festugière (1973, p. 9 -16) y Goldschmidt (1971, p. 183), a su vez, manifiestan esta opinión. 
ejercicio purificador del alma que redunda provechosamente en la práctica $(69 \mathrm{a}-\mathrm{e})^{{ }^{24} \text {. }}$.

Respecto de lo mismo, el Fedón hace hincapié en que la sabiduría, al ser la primera virtud que otorga los lineamientos de las otras virtudes, requiere de la inteligencia para su ejercicio; estas últimas virtudes se constituyen, por lo tanto, como virtudes sociales o políticas que se aprenden por imitación o siguiendo el ejemplo de otros.

\section{4.- Escatología, areté y Psiché.}

Una vez instalados en el mito escatológico del Fedón (107 c - 115 a), Sócrates nos expone que al demostrarse la inmortalidad del alma, ésta no presentará ninguna otra salida de sus vicios más que el hacerse más sensata y mejor, en definitiva, virtuosa. Por esto, el criterio para la obtención de la virtud ascética del Fedón es la pureza ( $\kappa \alpha \theta \alpha \rho o ́ \tau \eta \tau \alpha)$, tal como queda reflejada en la bella lejanía que plantea Platón de nosotros respecto de la vista, el oído y la inteligencia (Fedón, 111 b).

A propósito de esto, A. E. Taylor (1977, p. 177) propone que el objeto inmediato y principal de toda la conversación es la justificación de la vida como 'tendencia del alma', al insistir sobre la divinidad del alma humana, y la 'imitación de Dios' (en tanto divinidad), como regla de derecho y razonable de conducta; la inmunidad de la vida frente a la muerte es una mera consecuencia, aunque una consecuencia importante de esta divinidad inherente. Tal como destaca Taylor, el argumento es, en el sentido propio de la palabra, de carácter moral; el valor y la dignidad del alma dan motivos razonables para esperar que la muerte de un hombre virtuoso sea la puerta de entrada a una vida mejor, una 'aventura' que pueda enfrentar con buen ánimo, siendo esto el resumen de todo el discurso dado por el propio Sócrates en el pasaje 114 d - 115 a del Fedón ${ }^{25}$.

De esta manera, es preciso hacer todo lo necesario para participar de la prudencia y la virtud en esta vida. A propósito de las almas y su destino, ya tratados en el mito escatológico de Gorgias (523 a y ss.) en un primer momento y complementado hasta el detalle en el mito de Er de República (X, 614 b - 621 d), Sócrates concluye la

\footnotetext{
${ }^{24}$ Además, la autora defiende esta tesis en otro de sus trabajos: "Lógos, inmortalidad y praxis en el Fedón”, en Kleos, Revista de Filosofia Antiga (2000), v. 4, n. 4: 155 - 181.

${ }^{25}$ Cf. H. G. Gadamer (1999 (b); p. 56) quien destaca en este sentido: "El destino futuro de los fallecidos tiene que depender de la moralidad de la vida que se haya vivido; ése es, al fin el resultado del entero dialogo".
} 
temática de la vida del filósofo, una vida en que se debe anhelar la virtud y despojarse del vicio, prescindiendo del cuerpo y aferrándose al alma, manifestando admiración a la sabiduría mas no a los placeres corporales que nos entierran en el reino de lo mutable (Fedón, $114 \mathrm{~d}-115$ a):

"debe estar confiado respecto de su alma todo hombre que en su vida ha enviado a paseo los demás placeres del cuerpo $(\sigma \tilde{\omega} \mu \alpha)$ y sus adornos, considerando que eran ajenos y que debía oponerse a ellos, mientras que se afanó por los del aprender ( $\mu \alpha v \theta$ óvevv), y tras adornar su alma no con un adorno ajeno, sino con el propio

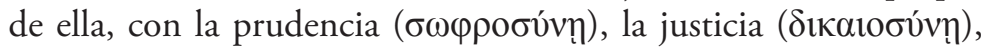

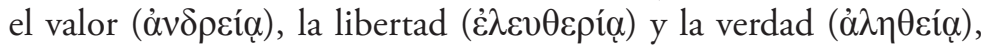
así aguarda el viaje hacia el Hades, como dispuesto a marchar en

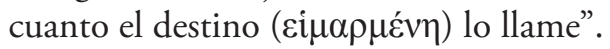

\section{Reflexiones finales}

A propósito de nuestro desarrollo, cabe destacar los siguientes aspectos que resultan, esperamos, iluminadores para futuras lecturas de Platón, y particularmente del Fedón:

a) El término kátharsis presenta al menos dos sentidos en el Fedón: el primero, y requisito ineludible del segundo, es aquel que precisa el aspecto 'purificador' de la virtud ascética del filósofo, a través de la cual éste se acerca tímidamente al conocimiento de la realidad; un segundo sentido corresponde principalmente a la kátharsis en tanto 'liberación' del alma respecto del cuerpo y a su conexión directa con el mundo eidético platónico.

b) La kathársis del Fedón sigue presente en la República aunque de un modo distinto, puesto que ya obteniendo la tripartición del alma, insigne logro antropológico de la Politeia platónica, el hombre ahora se ve inserto en la polis con su cuerpo ${ }^{26}$ y no aspirando a estar fuera de él. No obstante, sigue presente

${ }^{26}$ El alma, magnificada al máximo en el discurso del Fedón, necesita del cuerpo tal como se refleja en la República, puesto que a través de una educación rigurosa, particularmente en el ámbito de la gimnasia, se hace factible la consecución de una salud perfecta que implique el ejercicio filosófico. No obstante, nunca hay que perder de vista que es el alma la que predomina en la conjunción que conforma el ser humano junto al cuerpo. Wilamowitz (Plato I, p. 467) brinda una aproximación similar a propósito del mito del carro alado del Fedro (246 a - 248 c): "La complicada imagen del carro del alma con los dos caballos de distinto ánimo se ha pensado solo para el comportamiento del alma en el cuerpo humano". 
el anhelo por la consecución de la sabiduría, lo que inevitablemente le lleva a la búsqueda de las Ideas, objeto primero de las almas humanas. De hecho, esto se vuelve a resaltar con mayor claridad en el Filebo, diálogo en que se manifiesta que la vida buena debe consistir en aquellos placeres puros del alma.

c) Podemos desprender que existe una continuidad en lo que respecta al saber y su preponderancia para la conformación de la vida teórica y práctica del Fedón a la República, aun cuando también se manifiesta una contrariedad en cómo se entienden las virtudes, exceptuando la sabiduría: valentía, templanza y justicia son entendidas como virtudes de índole principalmente práctico, mientras que la sabiduría de orden teórico, no obstante también participa de la acción moral in situ (en tanto sabiduría práctica del orden moral [ $\varphi \rho o ́ v \eta \sigma ı \varsigma])$. Virtudes como la valentía, la temperancia y la justicia sólo se conforman como teóricas desde su formulación en el libro IV de la República.

d) En el Fedón, el alma es en sí misma phrónesis o sophía ('pensamiento puro'), representando ambas lo puro, conformando así, posteriormente, la parte superior del alma (logistikón), tal como se expresa en el planteamiento de la República, en donde Platón usa ambos términos indistintamente (phrónesis, IV, 433 b 8; sophia, IV, 443 e 6). En ese sentido, es preciso resaltar que el ejercicio de la phrónesis tiene como meta alcanzar las ideas, y así mismo, el conocimiento de lo real.

e) La vida es entendida como preparación para la muerte y la muerte como un fenómeno positivo para la consecución de la sabiduría y del encuentro con las Ideas, fin necesario para la obtención de la virtud ascética, ideal propuesto en el Fedón.

f) Sócrates se perfila como ideal de la $\dot{\alpha} \sigma \kappa \eta \tau \imath \kappa \tilde{\eta} \varsigma ~ \grave{\alpha} \rho \varepsilon \tau \tilde{\eta} \varsigma$ al morir por sus convicciones sobre el saber y el accionar moral y político de la polis, asumiendo la cicuta como camino necesario de coherencia y conocimiento, único camino para alcanzar, en forma efectiva y real, el conocimiento eidético sobre el orden cósmico y moral ${ }^{27}$.

${ }^{27}$ En la Historia del Arte se hace presente la obra del pintor Jacques Louis David, La Muerte de Sócrates (1787), máximo representante del neoclasicismo francés, que pese a las inexactitudes históricas, muestra cómo el fundador de la Ética se dispone a beber la cicuta en sus momentos finales de vida terrenal, debido a la injusticia cometida por sus quisquillosos detractores. Dicha pintura manifiesta una fuerte carga simbólica, al destacar con vehemencia y serenidad la aceptación de la muerte por parte del filósofo condenado.

Otro precursor del neoclasicismo en Francia fue Jean-François-Pierre Peyron, quién también inmortalizo la última escena socrática en 1787, paradójicamente en el mismo año en que fue 
El epílogo del diálogo (Fedón, 118 c) muestra esta idea. Una vez que Critón le cerró los ojos y la boca a Sócrates, Fedón le dijo a Equécrates: "Éste fue el fin...que tuvo nuestro amigo, el mejor hombre, podemos decir nosotros, de los entonces

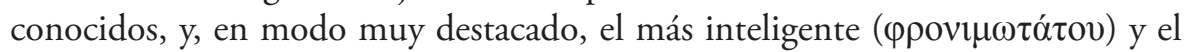

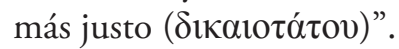

realizado el trabajo de David. Otros franceses que expresaron la triste escena fueron: Charles Alphonse du Fresnoy, quien cuenta con una obra que data del año 1650 aproximadamente, y Jacques-Philip-Joseph de Saint-Quentin, que en 1762 registró la muerte del maestro de Platón. En 1872, el pintor peruano Daniel Hernández ofreció al desarrollo del arte La muerte de Sócrates, pieza que le valió el primer reconocimiento de su carrera. 


\section{Bibliografía}

Astius, F. (1838): Lexicon platonicum sive vocum platonicarum Index [3 T.]. Libraria Weidmanniana: Lipsiae.

Bailly, A. (1950): Dictionnaire Étymologique Grec-Francais. Libraire Hachette: París.

Bluck, R. S. (1955): Plato's Phaedo. Routledge \& Kegan Paul: London.

Bonitz, .H (1867): Zu Platons Phädon 62 A, Hermes, 2. Bd., H. 2, pp. $307-$ 312. URL: http://www.jstor.org/stable/4471022

Brun, J. (1981): Platón y la Academia. Editorial universitaria de Buenos Aires: Argentina.

Chantraire, P. (1968 - 77): Dictionnaire étimologique de la langue grecque. Histoire des mots [4 t.]. Klincksieck: París.

Cornford, F. M. (2004): From religion to philosophy. A Study in the Origins of Western Speculation. Dover Publications, Inc. Mineola, New York.

Des Places, E. (1964): Lexique de la langue philosophique et religieuse de Platon [2 T.]. Les Belles Letres: París.

M. Dixsaut (1991): Phédon. Traduction, introduction et notes par M. Dixsaut. Flammarion: Paris.

Eggers Lan, C. (1971): El "Fedón” de Platón. Eudeba: Buenos Aires.

Festugière, A. (1973): Les trois protreptiques de Platon, Euthydème, Phédon, Epinomis. Vrin: Paris.

Paris.

(1975): Contemplation et vie contemplative selon Platon. Vrin:

Gadamer, H. G. (1980) "The Poofs of Inmortality in Plato's Phaedo", en Dialogue and Dialectic: Eight Hermeneutical Studies in Plato, Univ. Yale P., 21-38: New Haven.

(1999): (a) "El terreno sólido: Platón y Aristóteles” y (b) "Vida y alma (Fedón)", en El inicio de la filosofía occidental. Paidos: Barcelona.

Gioia, F. G. (2000): Lógos, inmortalidad y praxis en el Fedón, en Kleos, Revista de Filosofía Antigua, v. 4, n. 4, Rio de Janeiro. URL: http://academia. edu/1633911/LOGOS_INMORTALIDAD_Y_PRAXIS_EN_EL_FEDON 
(2009): Examen, mito y tradición en el Fedón. Una invitación a filosofar, en Correa Motta, A. y Zamora, J. M. (eds.), Eúnoia: Estudios de filosofía antigua: un homenaje a Maria Isabel Santa Cruz, Bogotá. URL: http://academia. edu/1633949/_Examen_mito_y_tradicion_en_el_Fedon._Una_invitacion_a_ filosofar.

Goldschmidt, V. (1971): Les Dialogues de Platon: Structure et Méthode dialectique. PUF: París.

París.

(1985): Le paradigme dans la dialectique platonicienne. J.Vrin:

Gomperz, T. (2000): Pensadores griegos. Tomo II, Sócrates y Platón. Herder. Barcelona.

Grube, G. M. A. (2010): El pensamiento de Platón. Gredos: Madrid.

Guthrie, W. K. C. (1998): Historia de la Filosofía griega. Tomo IV, Platón: El hombre y sus diálogos: primera época. Gredos: Madrid.

Hackforth, R. (1955): Plato's Phaedo. Cambridge University Press: Cambridge.

Jaeger, W. (2006): Paideia: los ideales de la cultura griega. Fondo de Cultura Económica: México.

(2003): La teología de los primeros filósofos griegos. Fondo de Cultura Económica: México.

Joly, H. (1980): Le renversement platonicien. Logos, episteme, polis. J. Vrin: París.

Pabón S. de U., José M. (2009): Diccionario Vox Manual Griego - Español. Bibliograf: Barcelona.

Platón (1999 - 2006): Diálogos. Traductores varios [9 vol.]. Editorial Gredos: Madrid.

(1889 - 1995): Platonis Opera. J. Burnet y otros editores, [5 Vol.]. Oxford University Press: Oxford.

Pohlenz, M. (1913): Aus Platos Werdezeit. Weidmannsche Buchhandlung: Berlín.

Robin, L. (1935): Platon. PUF: París.

(1962): El pensamiento griego y los orígenes del espiritu científico.

UTEHA: México. 
Schunl, P - M. (1956): La obra de Platón. Hachette: Buenos Aires.

Steiner, A. (1916): Die Etymologien in Platons Kratylos, Archiv für Geschichte der Philosophie, vol. 29, n 2, p. 109 - 132. URL: http://www.degruyter.com/ view/j/agph.1916.29.issue-2/agph.1916.29.2.109/agph.1916.29.2.109.xml

Stewart, H. (1915): Was Plato an ascetic?, The Philosophical Review, vol. 24, No 6, p. 603 - 613. URL: http://www.jstor.org/stable/2178212

TaYlor, A. E. (1946): El platonismo y su influencia. Nova: B. Aires. (1977): Plato. The man and his work. Methuen: London.

Velásquez, O. (2005): 'A $\lambda \hat{\theta} \theta \varepsilon 1 \alpha$ y sus usos y significados en Platón, Revista Iter, ¿Quid est veritas?, pp. 49-59. URL: http://www.diadokhe.cl/media/ platonica/Aletheia.pdf 